\title{
Characteristics of the Termination Shock: Insights from Voyager
}

\author{
A. C. Cummings and E. C. Stone \\ California Institute of Technology, Mail Code 220-47, Pasadena, CA 91125, USA
}

\begin{abstract}
We examine the energy spectra obtained from the cosmic ray instrument on the Voyager 1 spacecraft during 2002/215 through 2005/60. We find that the energy spectra of protons below $\sim 20 \mathrm{MeV}$ often resemble two power laws with a relatively hard index at low energies and a softer index at higher energies. The point of intersection of the two power laws is $\sim 3 \mathrm{MeV}$. Beginning in 2005, the low-energy index is typically -1.5 , corresponding to a shock strength (compression ratio) of 2.5. We attribute these characteristics to a restricted region of the solar wind termination shock that is sporadically connected to the Voyager 1 spacecraft by the interplanetary magnetic field. The absence of significant spectral variability in 2005 suggests that Voyager 1 entered a region with minimal spatial gradients of the lowest energy ions.
\end{abstract}

Keywords: Anomalous cosmic rays, solar wind termination shock, Voyager

PACS: $96.40 . C d, 96.40 . \mathrm{De}, 96.40 . \mathrm{Kk}, 96.50 . \mathrm{Pw}$

\section{INTRODUCTION}

Since mid-2002 the charged particle instruments on the Voyager 1 (V1) spacecraft have been detecting very large intensities of low-energy particles. The first episode of these enhanced intensities lasted approximately six months ${ }^{1,2}$. The particles are believed to be coming from the termination shock, presumably from the sunward side of the shock since at the time of the first intensity enhancement in mid-2002 the magnitude of the magnetic field did not show the enhancement expected if the termination shock were crossed ${ }^{3}$. There is often significant beaming of the particles outward along the azimuthal magnetic field that occurs sporadically as the scattering mean free path varies along the field line connecting Voyager 1 to the particle source.

The solar wind termination shock is the likely source of anomalous cosmic rays (ACRs), which originate as interstellar neutral atoms ${ }^{4}$ that become ionized in the heliosphere and carried to the shock where they are accelerated to cosmic ray energies ${ }^{5}$. The region of the shock connected to Voyager 1 is apparently not the source of ACRs, as the ACR energy spectra are strongly modulated during the periods of enhanced intensity at low energies and show little variability ${ }^{2}$. However, the lowenergy part of the energy spectrum of the new low-energy component often appears to have the power-law shape expected from diffusive shock acceleration ${ }^{6}$. The power-law dependence usually extends up to $\sim 3 \mathrm{MeV}$, where the spectrum rolls off into a steeper power law. In this paper, we examine these two properties of the energy spectra of $\mathrm{H}$ 
for data acquired from the Cosmic Ray Subsystem (CRS) instrument ${ }^{7}$ on Voyager 1 from $2002 / 215$ to $2005 / 60$.

\section{ANALYSIS TECHNIQUE}

The Voyager CRS instruments on each of the Voyager 1 and 2 spacecraft have 9 telescope apertures for recording cosmic rays of various types in several energy bands. The relevant telescope bore sights are shown in Fig. 1 for day 300 of 2002. The directions change little over the period of analysis reported here. The designations A, B, C, and D refer to four essentially identical Low-Energy Telescopes (LETs), which provide the energy spectra of $\mathrm{H}$ from $0.5-7.8 \mathrm{MeV}$, He from 1.0-7.8 MeV/nuc, and $\mathrm{O}$ from 1.05-17.1 MeV/nuc. The bore sights of the High Energy Telescope (HET) apertures are also shown. The HET telescopes (H1 and $\mathrm{H} 2)$ cover the energy ranges from 7.5-375 MeV for $\mathrm{H}, 7.5-540 \mathrm{MeV} /$ nuc for $\mathrm{He}$, and 11.9-125 MeV/nuc for $\mathrm{O}$. Only the $\mathrm{H} 2$ telescope is used for $\mathrm{H}$ and $\mathrm{He}$ analysis and a combination of both $\mathrm{H} 1$ and $\mathrm{H} 2$ is used for $\mathrm{O}$.

Streaming of 3.3-7.8 MeV protons along the azimuthal direction is evident in the lower panel of Fig. 2. The ratio of the average intensity in LET A and LET B to the intensity in LET $\mathrm{C}$ telescope is mostly $>1$ during the periods of enhanced intensity, indicating flow outward along the nominal azimuthal magnetic field direction. Note the intensity of ACR O is relatively steady throughout the period.

Also shown in Fig. 2 are the energy spectrum for protons observed at Voyager 1 for 2004 day 169 (92.2 AU and $34 \mathrm{~N}$ ) and spectra of helium and oxygen for the period 2004/163-2004/175. The He spectrum shows three components: the new low-energy component, the ACR component with a peak energy at $\sim 30 \mathrm{MeV} /$ nuc, and the galactic
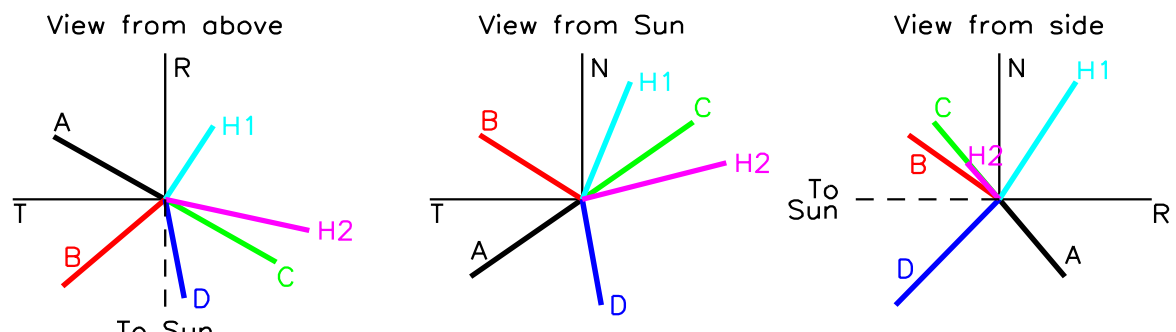

To Sun

FIGURE 1. View directions of the telescope apertures of the Voyager CRS instrument for 2002 day 300 in the RTN coordinate system as described in the text. The RTN coordinate system is spacecraft centered with $\mathrm{R}$ pointed radially away from the Sun, $\mathrm{T}$ is counterclockwise in the azimuthal direction, and $\mathrm{N}$ completes the right-handed coordinate system. 

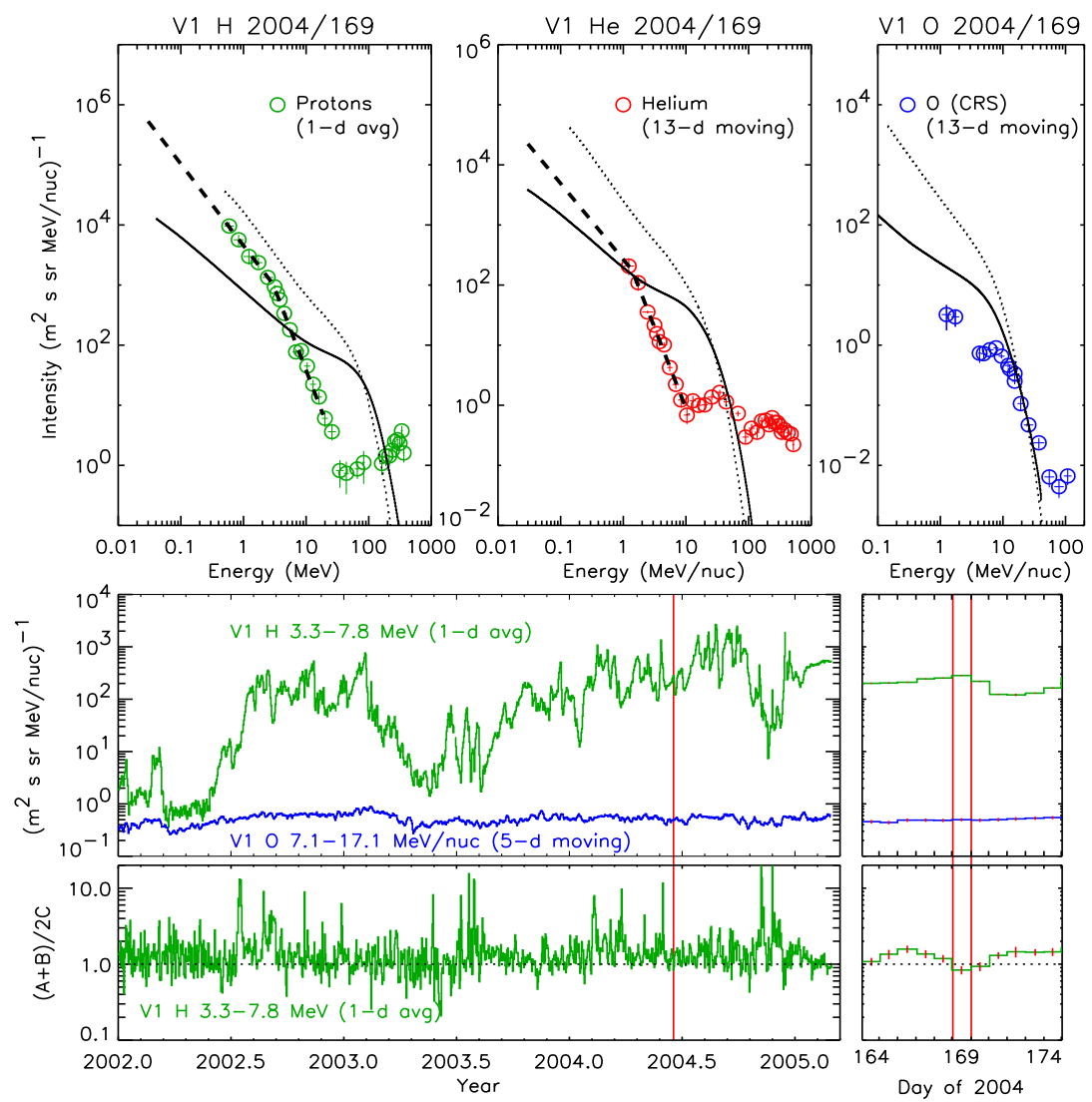

FIGURE 2. (top panels) Energy spectra of H, He, and O from V1 for the period indicated. The LET H and He points from 3.3-7.8 MeV and 3.0-7.8 MeV/nuc, respectively, are from the LET D telescope only. At lower energies for $\mathrm{H}$ and $\mathrm{He}$ and below $2 \mathrm{MeV} /$ nuc for $\mathrm{O}$, the data are from a single-parameter analysis of the first detector in the LET A telescope. The $\mathrm{H}$ points in this energy range have been corrected to the LET D direction by the ratio of the LET D to LET A intensity in the 3.3-7.8 MeV interval. The single parameter He and $\mathrm{O}$ points are also corrected to the LET D direction using the LET D to LET A intensities in the 3.0-7.8 MeV/nuc He interval. The dashed lines are fits to a two-power-law function described in the text. The solid and dotted lines are energy spectra expected at the termination shock as described in the text. (middle panel) Intensity of 3.3-7.8 MeV protons and 7.1-17.1 MeV/nuc ACR O vs time. (bottom panel) Azimuthal streaming index as described in the text. At the right in the bottom two panels, corresponding data for an 11-day period centered on 2004/169 are shown.

cosmic ray (GCR) component, which peaks at $\sim 250 \mathrm{MeV} /$ nuc. The dashed line in the $\mathrm{H}$ panel represents a fit of the low-energy data to a function consisting of two power laws, intersecting at a rolloff energy Eo_H. In the helium panel, the dashed line is similar except the power-law index at low energies is fixed by the fit to the $\mathrm{H}$ data. 
Also shown for reference are spectra for ACRs expected at the termination shock for both a strong shock $(r=4)$ and a weak shock $(r=2.4)^{8,9}$. The differences in the spectra of the low-energy and ACR components indicate that they come from different source regions on the shock. The source of the low-energy particles is connected to V1 along the magnetic field while the source of the ACRs, which are higher energy, is from a region that is not magnetically connected to Voyager.

In Fig. 3 we show the four parameters from a least-squares fit of a two-power-law function to the $\mathrm{H}$ spectra from $0.5-22.3 \mathrm{MeV}$ for those days from 2002/215 to 2005/60 where the estimated background in the single-parameter $\mathrm{H}$ data was less than $60 \%$. The top panel shows the intensity at $0.5 \mathrm{MeV}$ from the fit, together with the observed 3.3-7.8 MeV $\mathrm{H}$ intensity in LET D. The low-energy power-law index, a_H, shows relatively little change throughout the 2.5 year period, occupying a band principally between $\sim-1$ to $\sim-1.7$. The high-energy power-law index, b_H, shows more variability, ranging mainly from $\sim-2$ to -4 . The rolloff energy remains remarkably steady with a typical value of $\sim 3 \mathrm{MeV}$. Beginning in early 2005 , the energy spectra show much less daily variation than during 2002.5-2005.0, suggesting that Voyager 1 entered a region
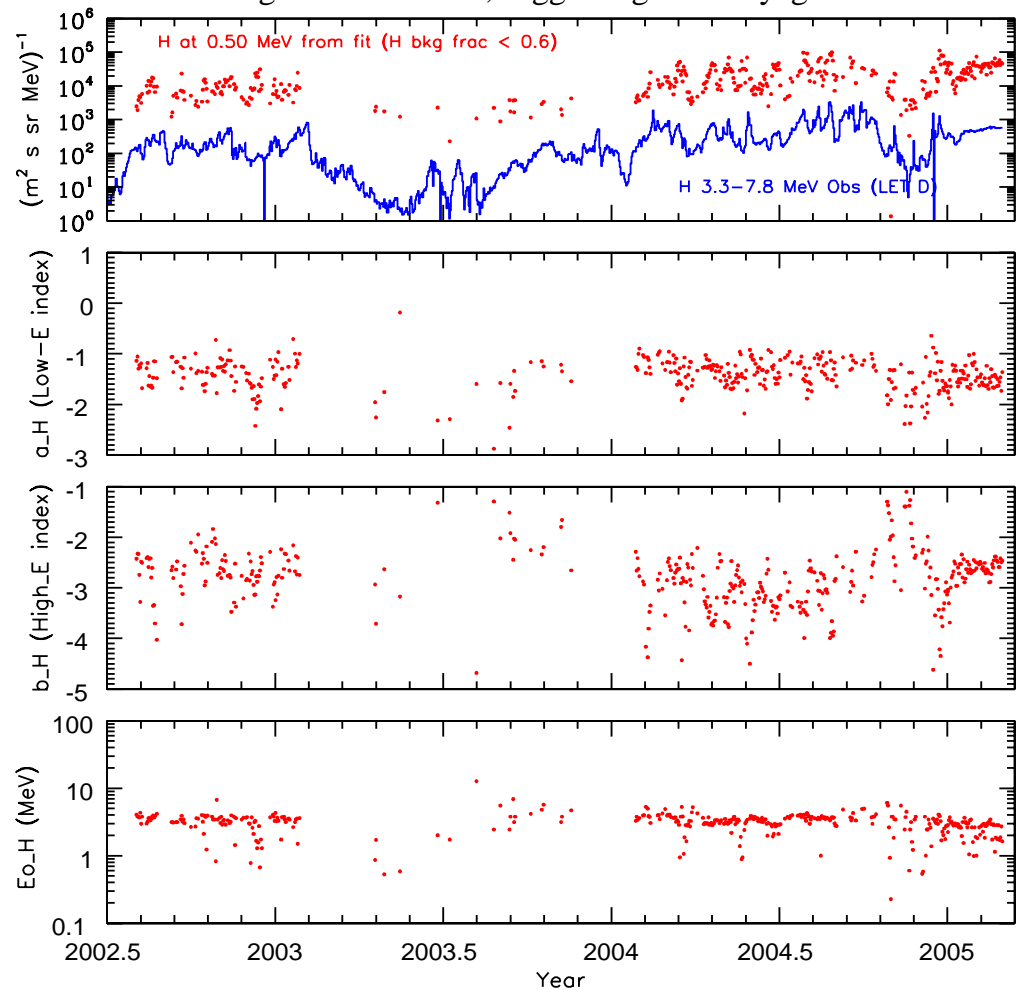

FIGURE 3. (top panel) Intensity of 3.3-7.8 MeV protons from V1 LET D and the intensity of 0.5 MeV from the fit to the two-power-law function vs time. (bottom panels) Low-energy power-law index $($ a_H $)$, high-energy power-law index $($ b_H $)$, and rolloff energy $($ Eo_H) from the model fits vs time. 
with minimal spatial gradients of the low-energy ions. This new regime will be discussed in a future paper.

\title{
DISCUSSION
}

The energy spectra shown in Fig. 2 are but one example of many spectra during the period 2002/215-2005/60 which appear to be spectra somewhat similar to those expected from diffusive shock acceleration (see reviews by Drury ${ }^{10}$ and Jones and Ellison $\left.{ }^{11}\right)$. The power-law dependence at low energies is directly related to the compression ratio at the shock (see, e.g., Blandford and Ostriker $\left.{ }^{6}\right)$ : $a \_H=(r+2) /(2-2 r)$, where a_H is the power-law index and $\mathrm{r}$ is the compression ratio (or shock strength). In 2005, the low-energy power-law index in Fig. 3 is typically -1.5, implying a shock with a compression ratio of 2.5. We attribute the spread in indices in prior years to modulation of the source spectrum by turbulence between the observation point and the source.

Numerical models of the spectrum of ACR $\mathrm{H}$ at the termination shock generally show an exponential rolloff in intensity at $\sim 100 \mathrm{MeV}$ (see, e.g., Cummings et al. ${ }^{8}$ ). Florinski and Jokipii ${ }^{12}$ attribute the rolloff to either cooling in the supersonic solar wind, particle escape across the heliopause, or curvature of the shock seen by particles in the heliosheath. In a non-equilibrium situation, the acceleration time scale would also limit the maximum energy in the accelerated energy spectrum. The rolloff energy of the low-energy component is only $\sim 3 \mathrm{MeV}$ and shows no upward trend over the approximately 2.5 years of the observations. Hence, the part of the shock producing these particles appears to be stable over a 2.5 -year period. We also do not observe an exponential drop off in intensity above this rolloff energy, but rather a transition to another power-law dependence. These aspects of the energy spectra need further theoretical study.

\section{ACKNOWLEDGMENTS}

This work was supported by NASA under contract NAS7-03001.

\section{REFERENCES}

\author{
S. M. Krimigis, R. B. Decker, M. E. Hill et al., Nature 426 (6962), 45 (2003). \\ Frank B. McDonald, Edward C. Stone, Alan C. Cummings et al., Nature 426 (6962), 48 (2003). \\ L. F. Burlaga, N. F. Ness, E. C. Stone et al., Geophysical Research Letters 30 (20), 2072 (2003). \\ L. A. Fisk, B. Kozlovsky, and R. Ramaty, Astrophys. J. Lett. 190, L35 (1974). \\ M. E. Pesses, J. R. Jokipii, and D. Eichler, Astrophys. J. Lett. 246, L85 (1981). \\ R. D. Blandford and J. P. Ostriker, Astrophys. J. Lett. 221, L29 (1978). \\ E. C. Stone, R. E. Vogt, F. B. McDonald et al., Space Sci. Rev. 21, 355 (1977). \\ A. C. Cummings, E. C. Stone, and C. D. Steenberg, Astrophys. J. 578, 194 (2002). \\ A. C. Cummings, E. C. Stone, and C. D. Steenberg, Astrophys. J. 581, 1413 (2002). \\ L. Drury, Rep. Prog. Phys. 46, 973 (1983). \\ F. C. Jones and D. C. Ellison, Space Sci. Rev. 58, 259 (1991). \\ V. Florinski and J. R. Jokipii, Astrophys. J. 591, 454 (2003).
}

